\title{
Fluorescence imaging assisted by surface modes on dielectric multilayers
}

\author{
Emiliano Descrovi ${ }^{1}$, a, Davide Morrone ${ }^{1}$, Angelo Angelini ${ }^{1,2}$, Francesca Frascella ${ }^{1}$, Serena Ricciardi ${ }^{1}$, Paola Rivolo $^{1}$, \\ Natascia De Leo ${ }^{2}$, Luca Boarino ${ }^{2}$, Peter Munzert ${ }^{3}$, Francesco Michelotti ${ }^{4}$, and Fabrizio Giorgis ${ }^{1}$ \\ 1 Dipartimento di Scienza Applicata e Tecnologia, Politecnico di Torino, C.so Duca degli Abruzzi 24, 10129 Torino, Italy \\ 2 Nanofacility Piemonte, Istituto Nazionale di Ricerca Metrologica, Strada delle Cacce 91, 10135 Torino, Italy \\ 3 Fraunhofer Institute for Applied Optics and Precision Engineering IOF, Albert-Einstein-Str. 7, 07745 Jena, Germany \\ 4 Dipartimento di Scienze di Base e Applicate per l'Ingegneria, SAPIENZA Università di Roma, Via A. Scarpa 16, \\ 00161 Roma, Italy
}

Received 27 August 2013 / Received in final form 28 December 2013

Published online 18 March 2014 - (C) EDP Sciences, Società Italiana di Fisica, Springer-Verlag 2014

\begin{abstract}
We propose a novel approach for wide-field fluorescence imaging based on dielectric multilayers on glass coverslides. It is demonstrated that the fluorescence radiated by emitters in proximity to the multilayer surface can couple to Bloch Surface Waves and subsequently leaks into the glass. The coupled fluorescence is beamed with low divergence according to the Bloch Surface Waves spectral/angular dispersion and then collected with a low-magnification imaging system. Since the coupling between emitter and surface modes is dependent on the surface local morphology, we find a strong spectral deformation of the collected fluorescence spectrum depending on the corresponding surface relieves imaged.
\end{abstract}

Modern optical sensing applications based on fluorescence take advantage of a number of photon-management strategies made available thanks to the progress in micro and nanofabrication [1]. In particular, the use of dispersive photonic structures allowing an angular separation of different spectral components of an emitted radiation is gaining an increasing popularity [2]. This effect can be obtained by providing engineered nanostructures having a suitable photonic density of states which the fluorescence radiated by an emitter close to the structure surface can couple in [3]. Probably, the most widely known example of spectral/angular control of emitted fluorescence is represented by the Surface Plasmon Coupled Emission (SPCE) occurring on flat and structured metallic films [4-6]. In this case, emitters located a few nanometers close to the metallic film can strongly modify their radiation pattern by virtue of a near-field coupling to surface plasmons. As a consequence of the energy and momentum conservation, fluorescence coupled to plasmonic modes can farfield radiate according to well defined spectral/angular dispersions [7].

Complementary to metallic structures, photonic crystals can be used for similar purposes. In this case, photonic modes for fluorescence coupling are provided by means of periodic arrangements of dielectric materials such as gratings [8] or multilayers [9]. In this scenario, photonic crystals provide several advantages with respect to surface plasmons. In fact photonic crystals exhibit a lower

\footnotetext{
a e-mail: emiliano.descrovi@polito.it
}

absorption (and corresponding higher resonance quality factors) in the visible range, spectral and polarization tunability and they offer the opportunity to exploit a wide range of surface functionalization chemistry for sensing $[10,11]$. Furthermore, beside the engineering of the fluorescence emission, photonic crystals can also be used for enhancing the fluorescence excitation $[9,12]$ eventually on a spatially multiplexed basis [13].

In this work, we use a one-dimensional photonic crystal (1DPC) sustaining TE-polarized Bloch surface waves (BSW) [14] in a fluorescence imaging setup using a prism mounting. 1DPC structures sustaining BSW can enhance and control the fluorescence emission of organic dyes located on a properly designed 1DPC surface $[15,16]$. It has been shown that the detected BSW-coupled fluorescence is strongly polarized and directional, with a small angular divergence and a corresponding narrow spectral bandwidth. Furthermore, a dielectric-loading mechanism similar to that for plasmons on smooth metallic films is such that significant red-shifts of the BSW-coupled fluorescence are produced depending on the local amount of the dielectric load on the 1DPC surface [17]. Here we exploit such an angular/spectral shift of BSW-coupled emission for demonstrating a colour multiplexed fluorescence imaging that is sensitive to the $1 \mathrm{DPC}$ surface relieves.

The photonic structure and the principle of detection are shown in Figure 1. A dielectric 1DPC constituted by 6 alternating layers of $\mathrm{Ta}_{2} \mathrm{O}_{5}$ (thickness $70 \mathrm{~nm}$ ) and $\mathrm{SiO}_{2}$ (thickness $210 \mathrm{~nm}$ ) is deposited on a thin glass substrate 


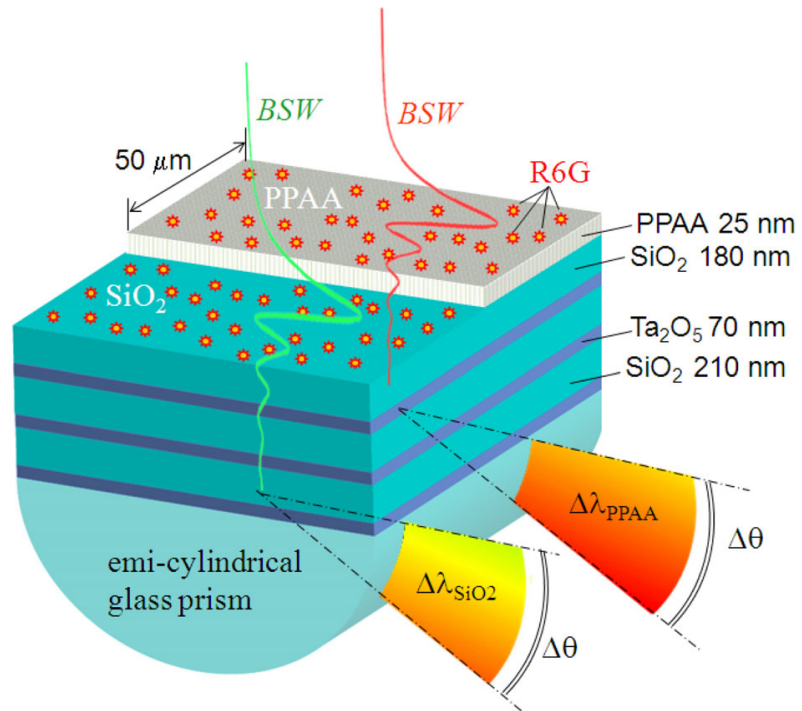

Fig. 1. Schematic view of the photonic structure and the detection configuration. R6G molecules are spun over the patterned 1DPC surface. BSW-coupled fluorescence is leaking through an emi-cylindrical prism and collected by a color CMOS-based imaging system including a $50 \mathrm{~mm}$ focal length lens and having a magnification factor of 1.7 .

that is oil-contacted to a glass prism. The last $\mathrm{SiO}_{2}$ layer has a thickness of $180 \mathrm{~nm}$. On the surface, a polymeric pattern constituted by a linear grating made of polyacrylic acid (PPAA) is plasma-deposited $[18,19]$ with a thickness of roughly $25 \mathrm{~nm}$ and a period of $100 \mu \mathrm{m}$. Finally, an ethanol solution containing Rhodamine $6 \mathrm{G}$ with a concentration of $1.2 \times 10^{-2} \mathrm{mg} / \mathrm{mL}$ is spun on the surface, and excited $(\lambda=532 \mathrm{~nm})$ by a weakly focused laser beam at normal incidence from the air side.

The 1DPC and the polymeric pattern are such that a pair of TE-polarized BSWs in the visible range can be sustained (electric field parallel to the 1DPC surface). More specifically, the BSW sustained on the PPAA ridges is redshifted with respect to BSW on the bare silica 1DPC surface. In such a configuration, when R6G molecules are excited, the fluorescence emission can couple to corresponding BSWs depending on their spatial location. This effect results in a BSW-coupled fluorescence leaking outside the glass prism with a well defined angular/spectral dispersion, i.e. a specific spectral band corresponding to a defined angular range of the outgoing radiation. Thanks to the unique features of spectral narrowness of BSW resonances, a well defined spectral component is preferentially detected at a given angle of detection. As shown in the following, this feature will allow a net colour discrimination of ridges with respect to trenches, despite the emitting molecules on the whole structure are of the same kind.

The spatially dependent coupling of fluorescence into the sustained BSWs is firstly investigated by means of a spectroscopic goniometric setup described in detail elsewhere [15], wherein an angularly resolved measurement of fluorescence spectra leaking out of the prism can be collected. In the present case, the fluorescence excitation is provided by means of a laser illumination incident normally from the air side of the structure. Measured fluorescence spectra are shown in Figure 2a for different angles of detection $\theta$, as indicated in the inset, with the grooves oriented parallel to the detection direction.

It is noted that TE-polarized narrow fluorescence peaks appear as the detection angle moves beyond the critical angle for a glass-air interface. In addition, these peaks are angularly distributed according to the BSW dispersion curve [15], therefore revealing the nature of BSW-coupled emission. For detection angles within a given range (comprised between $45.5 \mathrm{deg}$ and $48.5 \mathrm{deg}$ ), two peaks appears on the same spectrum. The two peaks are related to fluorescence coupled to BSW on the bare $\mathrm{SiO}_{2}$ 1DPC surface and the PPAA ridges, respectively. Due to the dielectric loading mechanism, the most redshifted peak belongs to $\mathrm{BSW}$ on PPAA ridges. Rigorous calculations validate this observation [17], confirming that the electric field distribution associated to those BSWs (e.g. calculated at a wavelength $\lambda=580)$ nm shows a preferential spatial localization either in the trenches or on the ridges (Figs. $2 \mathrm{~b}$ and 2c). Since the coupling of the fluorescence emission to surface modes occurs in a near-field regime, we can correlate the angular/spectral distribution of the detected BSW-coupled fluorescence with the spatial region wherein corresponding emitters are located (i.e. $\mathrm{SiO}_{2}$ trenches or PPAA ridges). In other words, if fluorescence imaging is performed through the glass prism under a given detection angle $\theta, \mathrm{R} 6 \mathrm{G}$ molecules located in spatial regions with different nanometric relieves can be imaged with different colours defined by a spectrally narrow BSW-coupled fluorescence.

When imaging is concerned, BSW-coupled leakage fluorescence is collected with a simplified imaging system employing a single collection lens with focal length $50 \mathrm{~mm}$ and a CMOS RGB camera. The overall magnification factor is estimated to be 1.73 , while the numerical aperture of the system is reduced to NA $=0.1$ by means of an aperture diaphragm. Illustrative collected images are presented in Figure 3.

Starting from a numerical aperture NA $=0.25$, we reduced to 11 degrees the full collection angle (corresponding to a $\mathrm{NA}=0.1$ ) by operating on a diaphragm aperture in front of the collection optics. Although the angular range of the collection optics is larger than the angular separation of the two BSWs, it has been possible to obtain a significant spectral selection of the BSW-coupled fluorescence by rotating the collection optics from 49.5 degrees to 45 degrees with respect to the $1 \mathrm{DPC}$ surface normal. Figure 3a relates to a collection angle $\theta \sim 49.5 \mathrm{deg}$. As it is possible to appreciate from Figure 2a, at this leakage angle, only the BSW on PPAA ridges can be detected. The resulting image shows PPAA ridges with a bright green-yellow colour. On the bottom part of the image, the normalized cross-sectional profiles of light intensity as seen by the $\mathrm{R}$ and $\mathrm{G}$ pixels of the CMOS camera are plotted. Here, fluorescence is detected by both the $\mathrm{R}$ and $\mathrm{G}$ channels, with a high contrast between PPAA ridges and $\mathrm{SiO}_{2}$ trenches. When the collection angle is decreased 

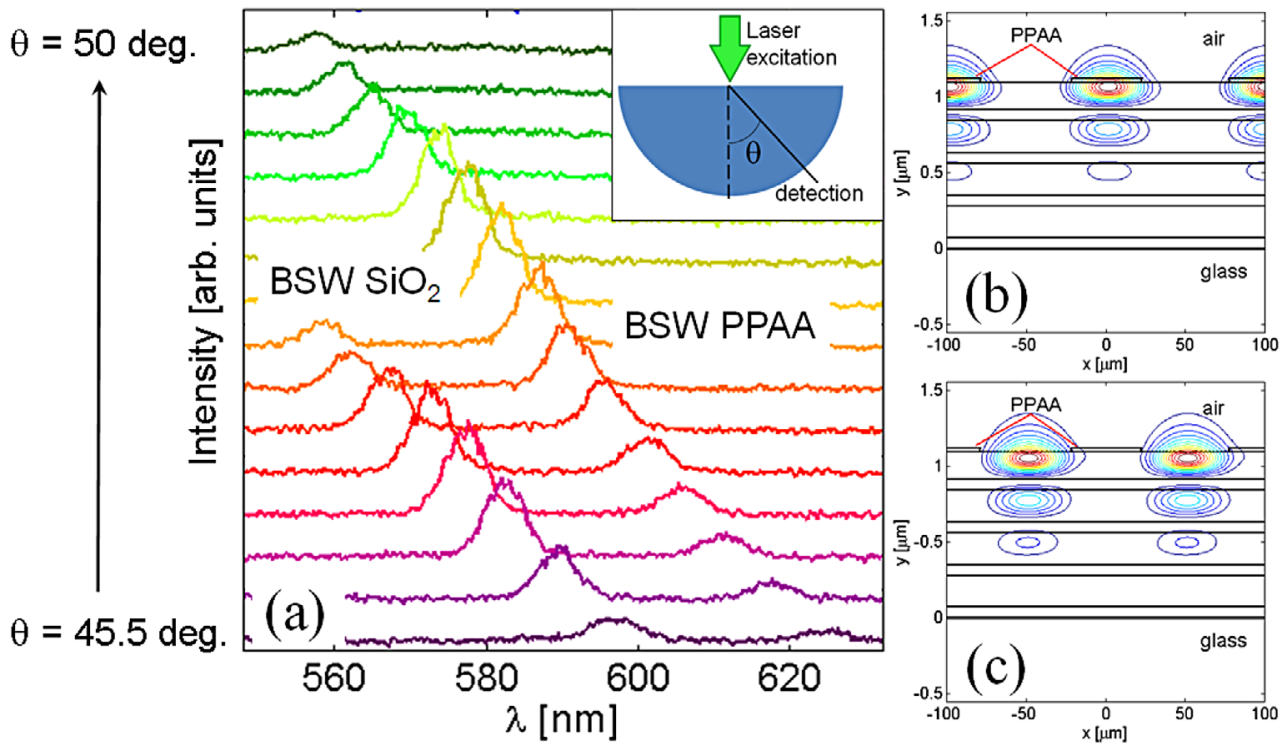

Fig. 2. Measured angularly-resolved fluorescence spectra leaking out the prism (inset: detection scheme) featuring two intensity peaks corresponding to BSWs on $\mathrm{SiO}_{2}$ trenches and PPAA ridges respectively (a); calculated electric field intensity distributions associated to either BSW at $\lambda=580 \mathrm{~nm}$ on PPAA ridges (b) or $\mathrm{BSW}$ on $\mathrm{SiO}_{2}$ trenches (c).
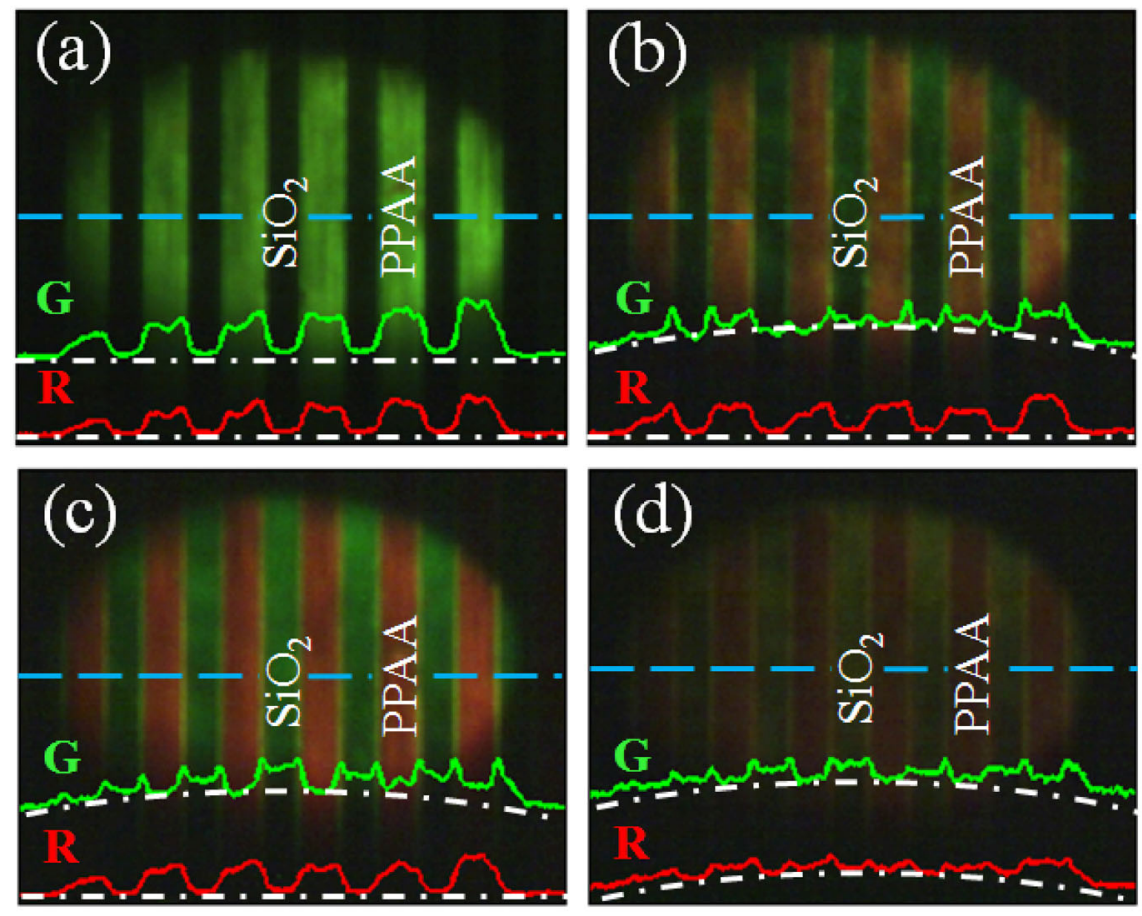

Fig. 3. BSW-coupled fluorescence images collected at collection angles: (a) $\theta \sim$ $49.5 \mathrm{deg}$; (b) $\theta \sim 48 \mathrm{deg}$; (c) $\theta \sim 46.5 \mathrm{deg}$; (d) $\theta \sim 45 \mathrm{deg}$. For each image, the normalized cross-sectional distribution of light along the cyan dashed line is shown on the bottom, as detected by the $\mathrm{R}$ and $\mathrm{G}$ pixels of the CMOS camera. The overall full collection angle of the imaging system in air is approximately $11 \mathrm{deg}$.

to $\theta \sim 48 \mathrm{deg}$ (Fig. $3 \mathrm{~b}$ ), the PPAA ridges start becoming more reddish and a greenish background belonging to $\mathrm{SiO}_{2}$ trenches is appearing. This effect produces a decrease of contrast of the light distribution on the $\mathrm{G}$ channel, while the $\mathrm{R}$ channel is still dominated by the contribution of the redshifted BSW associated to PPAA ridges. Here, BSWs related to $\mathrm{SiO}_{2}$ trenches are accessible at shorter wavelengths. At $\theta \sim 46.5 \mathrm{deg} \mathrm{SiO}_{2}$ trenches show of a bright green-yellowish colour, while the PPAA ridges are turned definitely to red (Fig. 3c). The G channel shows a dominant contribution coming from the $\mathrm{SiO}_{2}$ trenches, and the cross sectional profile is inverted with respect to the previous images. Nevertheless, the contrast is not high since the contribution from PPAA ridges is still present and this produces a smooth non-flat background of the signal on the $\mathrm{G}$ channel. In Figure 3d an image collected at $\theta \sim 45 \mathrm{deg}$ is shown. At this leakage angle, the fluorescence intensity coupled to BSWs is dramatically darker because the corresponding spectral regions belong to the tail of the R6G emission spectrum. Nevertheless it is still 
possible to appreciate an overall colour difference between trenches and ridges, although the contrast in both the $\mathrm{G}$ and $\mathrm{R}$ channels is reduced. This translates into a significant overlap of both BSW-coupled fluorescence spectra and the cross-sectional light distribution exhibits a smooth non-flat background for both $\mathrm{R}$ and $\mathrm{G}$ channels.

In conclusion, we demonstrated a far-field imaging approach taking advantage of an emission-coupling mechanism occurring on the surface of dielectric 1DPC sustaining BSW. Such a BSW-coupled emission produces a well defined beaming of the emitted fluorescence that allows an efficient detection even when using a low numerical aperture collection optics. Thanks to the high sensitivity of BSWs, slight local perturbations of the surface (e.g. the addition of dielectric materials with nanometric thickness) produces a spectral/angular shift of BSW that can be directly imaged as a colour change of the considered area. Contrary to Surface Plasmon Polaritons, that are generally characterized by spectrally large resonances, such a color change can be better appreciated because of the narrow spectral width of the BSW wherein fluorescence in coupled.

The presented approach is simple, flexible, robust and can be easily implemented in existing multi-array biosensing chips [20] with an expected improved performance for multiplexed detection [21]. However, in case twodimensional spot arrays are considered, a further surface structuration can be introduced in order to limit the unwanted effects produced by the long BSW propagation length. For example, BSW propagation along the detection direction can be inhibited by locally tuning the dielectric loading between adjacent rows of spots.

This research has received funding from the European Union Seventh Framework Program (FP7/2007-2013) under Grant agreement No. 318035 - Project BILOBA and from the Italian FIRB 2011 NEWTON (grant RBAP11BYNP).

\section{References}

1. J.R. Lakowicz, in Principles of Fluorescence Spectroscopy, 3rd edn. (Springer, New York, 2006)

2. S.H. Cao, W.P. Cai, Q. Liu, Y.Q. Li, Annu. Rev. Anal. Chem. 5, 317 (2012)
3. W.L. Barnes, J. Mod. Opt. 45, 661 (1998)

4. I. Gryczynski, J. Malicka, Z. Gryczynski, J.R. Lakowicz, Anal. Biochem. 324, 170 (2004)

5. J.R. Lakowicz, K. Ray, M. Chowdhury, H. Szmacinski, Y. Fu, J. Zhang, K. Nowaczyk, Analyst 133, 1308 (2008)

6. J.R. Lakowicz, I. Gryczynski, K. Aslan, C.D. Geddes, in Fluorescence Sensors and Biosensors, edited by R.B. Thompson (CRC Press, Boca Raton, 2005)

7. C.Y. Jun, K.C.Y. Huang, M.L. Brongersma, Nat. Commun. 2, 283 (2011)

8. N. Ganesh, I.D. Block, P.C. Mathias, W. Zhang, E. Chow, V. Malyarchuk, B.T. Cunningham, Opt. Express 16, 21626 (2008)

9. I. Soboleva, E. Descrovi, F. Giorgis, C. Summonte, A.A. Fedyanin, Appl. Phys. Lett. 94, 231122 (2009)

10. F. Frascella, S. Ricciardi, P. Rivolo, V. Moi, F. Giorgis, E. Descrovi, F. Michelotti, P. Munzert, N. Danz, L. Napione, M. Alvaro, F. Bussolino, Sensors 13, 2011 (2013)

11. K. Toma, E. Descrovi, M. Toma, M. Ballarini, P. Mandracci, F. Giorgis, A. Mateescu, U. Jonas, W. Knoll, J. Dostalek, Biosens. Bioelectron. 43, 108 (2013)

12. C.S. Huang, S. George, M. Lu, V. Chaudhery, R. Tan, R.C. Zangar, B.T. Cunningham, Anal. Chem. 83, 1425 (2011)

13. V. Chaudhery, C.S. Huang, A. Pokhriyal, J. Polans, B.T. Cunningham, Opt. Express 19, 23327 (2011)

14. J.A. Gaspar-Armenta, F. Villa, T. López-Ríos, Opt. Commun. 216, 379 (2003)

15. M. Ballarini, F. Frascella, F. Michelotti, G. Digregorio, P. Rivolo, V. Paeder, V. Musi, F. Giorgis, E. Descrovi, Appl. Phys. Lett. 99, 043302 (2011)

16. R. Badugu, K. Nowazcyk, E. Descrovi, J.R. Lakowicz, Anal. Biochem. 442, 83 (2013)

17. M. Ballarini, F. Frascella, N. De Leo, S. Ricciardi, P. Rivolo, P. Mandracci, E. Enrico, F. Giorgis, F. Michelotti, E. Descrovi, Opt. Express 20, 6703 (2012)

18. S. Ricciardi, R. Castagna, S.M. Severino, I. Ferrante, F. Frascella, E. Celasco, P. Mandracci, I. Vallini, G. Mantero, C.F. Pirri, P. Rivolo, Surf. Coat. Technol. 207, 389 (2012)

19. A. Sinibaldi, N. Danz, E. Descrovi, P. Munzert, U. Schulz, F. Sonntag, L. Dominici, F. Michelotti, Sens. Actuat. B. 174, $292(2012)$

20. E. Matveeva, J. Malicka, I. Gryczynski, Z. Gryczynski, J.R. Lakowicz, Biochem. Biophys. Res. Commun. 313, 721 (2004)

21. J. Gao, A.M. Sarangan, Q. Zhan, Opt. Lett. 37, 2640 (2012) 